\title{
Paradoxical Respiration: An indication for Adenotonsillectomy?
}

\author{
J.M. Weinberger and M. Gross*
}

Department of Otolaryngology / Head \& Neck Surgery Hadassah Hebrew-University Medical Center, Jerusalem, Israel

\begin{abstract}
Paradoxical breathing or "paradoxical inward rib cage movements" without frank sleep apnea is a clinical description of the "upper airway resistance syndrome". Upper airway resistance syndrome is thought to be a distinct entity and not just a part of the continuum of obstructive sleep apnea; paradoxical breathing in children appears to be a separate entity from the classic patient with marked adenotonsillar hypertrophy causing obstructive apnea. The treatment also differs. We present a case of paradoxical breathing in a child who resolved spontaneously over a two year period.
\end{abstract}

Keywords: Paradoxycal respiration, obstructive sleep apnea, upper airway resistance syndrome, adenotonsillectomy.

\section{INTRODUCTION}

Obstructive sleep apnea secondary to adenotonsillar hypertrophy in children is well known. The stereotype toddler presenting with snoring, head extension (to maintain a patent airway) and frequent movements during sleep is easy to diagnose. The consequence of apneas is reduced oxygen saturation and hypercapnea and the preferred treatment is anatomical correction of the mechanical obstruction (adenotonsillectomy). In contrast, patients with upper airway resistance syndrome (UARS) are "silent snorers"; they do not drop their oxygen saturation and still can suffer from functional somatic syndromes, especially fatigue, (potentially worse than moderate apneic patients). Conventional wisdom presently categorizes upper airway resistance syndrome as being more common than obstructive sleep apnea [1]. The treatment for UARS is also less well established; in children, the old "stand-by" of adenotonsillectomy is still the recommended therapy. We would like to present a case of upper airway resistance syndrome in a 5 year old that resolved spontaneously over a two year period.

\section{CASE REPORT}

A 5 year old boy with atopic dermatitis and mild asthma treated with Montelukast-Sodium was evaluated regarding difficulty breathing at night without snoring. On physical examination he had an intact nasal airway with mild tonsillar hypertrophy (grade 2). The hard and soft palates were normal. Blood tests revealed mild eosinophilia - 8\% (range 0$5 \%)$ and elevated $\operatorname{IgE}(76 \mathrm{IU} / \mathrm{cc})$. X-ray of the nasopharynx showed a small adenoid pad.

A polysomnographic sleep study recorded oxygen saturations maintained over $94 \%$ without apneas. REM sleep accounted for $14 \%$ of the sleep time. However, frequent paradoxical respiratory movements were indicative of excessive effort at ventilation.

A discussion ensued regarding the appropriate treatment, and in the absence of significant adenotonsillar hypertrophy a "wait-and-see" approach was recommended. Spontaneous resolution was noted clinically over the next two years.

\footnotetext{
*Address correspondence to this author at the Department of Otolaryngology / Head \& Neck Surgery Hadassah Hebrew-University Medical Center, Jerusalem, Israel; E-mail: drgrossm@hotmail.com
}

\section{DISCUSSION}

There is controversy whether "sleep disordered breathing" constitutes a simple continuum of different severities of mechanical obstruction or if there are really diverse and distinct entities that are presently thrown together under this title. Initially described in children in 1982, and labeled in 1993, it seems that upper airway resistance syndrome is a separate category from the better recognized sleep apnea syndrome $[2,3]$. It appears to belong to the functional somatic syndromes and its spectrum includes fibromyalgia, chronic fatigue syndrome, migraine-tension headaches, irritable bowel and TMJ syndrome in adults [4]. The absence of decreased oxygen saturation and sometimes even the cornerstone symptom of snoring makes a diagnostic challenge and requires a high index of suspicion. It is estimated that $1 \%$ of all patients undergoing sleep studies for hypersomnolence suffer from silent UARS [5]. The rigorous diagnostic criteria for the specific diagnosis of UARS require Apnea/Hypopnea Index $<5$, oxygen saturation $>92 \%$, and esophageal manometry to record negative intrathoracic pressures during respiratory related arousals and associated limitation of air flow [6]. The characteristic abnormality on EEG is "alpha-delta sleep", the intrusion of waking alpha rhythm into deep, slow wave sleep and the higher rate of cyclic alternating patterns in non-REM sleep [7]. Essentially, patients with UARS have periods of sleep associated with air flow limitation, increased respiratory effort and arousals [3]. However, practically it is not possible to routinely monitor children (or adults) with esophageal manometers. Sleep studies with normal oxygen saturation and the absence of apneas associated with paradoxical breathing are the clinical correlate of the rigorous definition of UARS. The preservation of sensory perception in the palates of patients with UARS in contrast to the permanently blunted responses of patients with obstructive apnea would tend to support a conservative approach in the UARS group and a more aggressive approach (i.e. surgery) in the apnea group [6]. The question of treatment remains open and although the "gold-standard" has traditionally been adenotonsillectomy there may be a subset of patients in whom there is no significant adenotonsillar enlargement and "tincture-of-time" may be more appropriate. The natural history of the disorder in children is poorly defined and spontaneous resolution is likely common in children in contrast to adults. The categorization of UARS within the "so- 
matic syndromes" works well in adults but is less useful in children. It is interesting to speculate how UARS may resolve in children. One wonders if skeletal flexibility may be a contributing factor and as the child grows and the cartilaginous airway stiffens, upper airway collapse may become less prominent. Knowing that there are no immediate irreversible repercussions may allow for more judicious use of surgery in the care of toddlers with UARS.

\section{REFERENCES}

[1] Guilleminault C, Krahmtsov A. Upper airway resistance syndrome in children: a clinical review. Semin Pediatr Neurol 2001; 8: 20715.

[2] Guilleminault C, Winkle R, Korobkin R, Simmons F. Children and nocturnal snoring: evaluation of the effects of sleep-related respira- tory resistive load and daytime functioning. Eur J Pediatr 1982; 139: 165-71

[3] Guilleminault C, Stoohs R, Clerk A, Cetel M, Maistros P. A cause of daytime sleepiness: the upper airway resistance syndrome. Chest 1993; 104: 781-87.

[4] Guilleminault C, Dave R. The symptoms and signs of upper airway resistance syndrome: a link to the functional somatic syndromes. Chest 2003; 123: 87-95.

[5] Kristo DA, Lettieri CJ, Andrada T, Taylor Y, Eliasson AH. Silent upper airway resistance syndrome: prevalence in a mixed military population. Chest 2005; 127: 1654-57.

[6] Bao G, Guilleminault C. Upper airway resistance syndrome-one decade later. Curr Opin Pulm Med 2004; 10: 461-67.

[7] Guilleminault C, Dave R. Upper airway resistance syndrome, insomnia, and functional somatic syndromes. Chest 2003; 123: 1214 